\title{
Quantum Pure State Tomography via Variational Hybrid Quantum-Classical Method
}

\author{
Tao Xin,,${ }^{1, *}$ Xinfang Nie, ${ }^{1}$ Xiangyu Kong, ${ }^{2}$ Jingwei Wen, ${ }^{2}$ Dawei Lu,,${ }^{1, \dagger}$ and Jun $\mathrm{Li}^{1, \ddagger}$ \\ ${ }^{1}$ Shenzhen Institute for Quantum Science and Engineering and Department of Physics, \\ Southern University of Science and Technology, Shenzhen 518055, China \\ ${ }^{2}$ State Key Laboratory of Low-dimensional Quantum Physics and Department of Physics, Tsinghua University, Beijing 100084, China
}

\begin{abstract}
To obtain a complete description of a quantum system, one usually employs standard quantum state tomography, which however requires exponential number of measurements to perform and hence is impractical when the system's size grows large. In this work, we introduce a self-learning tomographic scheme based on the variational hybrid quantum-classical method. The key part of the scheme is a learning procedure, in which we learn a control sequence capable of driving the unknown target state coherently to a simple fiducial state, so that the target state can be directly reconstructed by applying the control sequence reversely. In this manner, the state tomography problem is converted to a state-to-state transfer problem. To solve the latter problem, we use the closed-loop learning control approach. Our scheme is further experimentally tested using techniques of a 4-qubit nuclear magnetic resonance. Experimental results indicate that the proposed tomographic scheme can handle a broad class of states including entangled states in quantum information, as well as dynamical states of quantum many-body systems common to condensed matter physics.
\end{abstract}

\section{INTRODUCTION}

Quantum state tomography (QST) is the art of determining a quantum state from making measurements on a set of informationally complete observables [1]. It plays a vital role in many quantum information processing tasks, such as in characterizing an interested target quantum system or in estimating the performance of a quantum computing experiment. However, QST experiments are subject to several crucial challenges. First, reconstructing the full density matrix of a quantum system is highly demanding in the sense that the resources required grow exponentially with the system size. Second, the reliability of QST is limited by sensitivity to statistical noise and experimental errors. These issues impose great difficulties in the practical applications of QST even for modest-sized quantum systems [2-5]. It is hence important to develop a robust, precise, and easy-to-implement method for determining unknown quantum states.

Significant efforts have been devoted to improving the performance of QST [6-13], including self-guided QST, adaptive QST, QST via reduced density matrices (RDMs), and QST via trained neural networks. Self-guided QST considers tomography as a projection measurement optimization problem and finds the optimal solution by stochastic approximation algorithms, which is robust against the noises in characterizing states. Adaptive QST approach in which the choice of the next measurement depends on the previous measurement outcomes may be as impractical as traditional QST for larger system size [14-16]. QST via RDMs measures only the local RDMs to determine the global state so that QST is significantly simplified by reducing the measurement resources [17-20]. Measuring local RDMs are usually convenient on realistic physical setups. Recent researches show that machine learning methods, e.g., multi-layer trained neural work, are promising to recover

\footnotetext{
*xint@sustech.edu.cn

†1udw@sustech.edu.cn

$\ddagger$ lij3@sustech.edu.cn
}

target states efficiently from the local information via RDMs [21-25]. Yet, in principle, how to recover an unknown quantum state from its local RDMs is generally an unsolved problem [26].

Recently, there has been a growing interest in the variational hybrid quantum-classical (HQC) approach, which is regarded as a strategy to boost the efficiency of quantum computational tasks before quantum supremacy is achieved [27-30]. In this approach, a quantum computer works in conjunction with classical routines to maximally reduce the requirements for expensive quantum resources. The difficult part of the target problem is accomplished on a quantum computer, while the relatively easier part is done with a classical computer. HQC approach is a novel attempt versus full-quantum computation [31, 32]. It has found many successful applications ranging from quantum chemistry simulation [33, 34], quantum optimal control [35, 36], and quantum error correction [37] to quantum state diagonalization [38].

In this work, we propose an effective QST procedure based on the HQC approach. For an $n$-qubit pure state $\rho$ to be reconstructed, this procedure attempts to find a unitary process $\mathcal{C}(t)$ that drives the system from $\rho$ to $|\mathbf{0}\rangle\langle\mathbf{0}|$ with $|\mathbf{0}\rangle=|0\rangle^{\otimes n}$. Once such a unitary process is found, the unknown state is directly obtained as $\rho=\mathcal{C}^{\dagger}|\mathbf{0}\rangle\langle\mathbf{0}| \mathcal{C}$. In our framework, $\rho$ is assumed to be a quantum experimental state, e.g., the final state of a quantum computing experiment. Despite lacking information regarding how $\rho$ is realized, it should come from a tractable process in the sense that it is prepared from a polynomially-scaled quantum process. Intractable processes are not expected to be observed or realized in experiment, thus only low complexity states are experimentally accessible [39]. This implies that the optimal unitary trajectory that connects $\rho$ and $|\mathbf{0}\rangle=|0\rangle^{\otimes n}$ would not be unreasonably long. The unitary trajectory is realized through a parameterized controlled evolution $\mathcal{C}(b, t)$, where $b$ represents the set of control parameters. So, our procedure actually seeks an optimal control sequence. In this way, the state tomography problem converts to an optimal control problem. In searching for the optimal control sequence, the target function to be optimized is chosen to be the distance between $|\mathbf{0}\rangle\langle\mathbf{0}|$ and the actual final state. This 
distance can be easily estimated with a few measurements for many experimental platforms. In our construction, a trusted quantum computer replaces a classical computer to efficiently perform the evolution of the sequence $\mathcal{C}(b, t)$ and precisely estimate both target function and its gradient in each iteration. Classical computer takes charge of determining the search direction and the step size to update the control parameters $b$.

QST using the HQC approach is a promising and applicable technique for reconstructing quantum states in current physical setups. Here, we also present an experimental demonstration of QST via HQC on a 4-qubit NMR quantum processor. We basically consider two types of quantum states, namely dynamical states of quantum many-body systems and entangled states. In the experiment, we successfully drove such states to the ground state, and reconstructed them at high-quality, without involving performing informationallycomplete measurements. Therefore, the feasibility and the ability of our proposed HQC based-QST method has been confirmed.

\section{METHOD}

\section{A. Problem Setting}

To start, we first introduce the problem by describing the general control setting that we address. We consider an $n$ qubit spin system with internal Hamiltonian $\mathcal{H}_{0}$ and control Hamiltonian $\mathcal{H}_{c}$. Normally, $\mathcal{H}_{0}$ is a two-body local Hamiltonian with constant system parameters. $\mathcal{H}_{0}$ together with $\mathcal{H}_{c}$ provide the ability to engineer the system with full controllability. The control is realized by a time-dependent magnetic field, namely, $\mathcal{H}_{c}=\sum_{i=1}^{n}\left\{b_{x}(t) \sigma_{x}^{i}+b_{y}(t) \sigma_{y}^{i}\right\}$, with $\sigma_{x}, \sigma_{y}$, and $\sigma_{z}$ being the three Pauli matrices, and $b(t)=$ $\left\{b_{x}(t), b_{y}(t)\right\}$ being the control sequence to manipulate the dynamics of the quantum system. Consider the situation when the spin system processor finishes some quantum task we submit, the final state $\rho$ contains the useful information to be extracted, or when there is an unknown state $\rho$ for which we are unaware of its earlier evolution history. The problem is to reconstruct $\rho$.

The conventional QST method works as follows. For an $n$-qubit unknown quantum state $\rho$, one can decompose it in terms of the Pauli product operator basis as $\rho=$ $\sum_{i_{1}, . ., i_{n}=0}^{3} \gamma_{i_{1}, . ., i_{n}} \sigma_{i_{1}} \otimes \cdots \otimes \sigma_{i_{n}}$. Here, $\sigma_{0}=I, \sigma_{1}=\sigma_{x}$, $\sigma_{2} \stackrel{=}{=} \sigma_{y}$, and $\sigma_{3}=\sigma_{z}$. The coefficient $\gamma_{i_{1}, . ., i_{n}}$ is the projective component of $\rho$ in $\sigma_{i_{1}} \otimes \cdots \otimes \sigma_{i_{n}}$. The standard QST estimates all the coefficients from a series of quantum measurements. There are in total $4^{n}-1$ and $2^{n}-1$ coefficients to be determined for mixed states and pure states, respectively. It is obvious that the exponential growth in the number of experiments needed to measure all of these coefficients results in the difficulty in performing standard QST for large quantum systems.

\section{B. Our Variational HQC Approach}

Here, we show that, instead of using traditional QST, the state $\rho$ can be reconstructed via a variational learning procedure in an iterative way. We first search an optimal control sequence $b(t)$ to drive the unknown state $\rho$ to the ground state $|\mathbf{0}\rangle\langle\mathbf{0}|$ and then realize the reconstruction task simply as $\rho=\mathcal{C}(b)^{\dagger}|\mathbf{0}\rangle\langle\mathbf{0}| \mathcal{C}(b)$. We have to choose a reasonable fitness function $f(b)$ as a function of the parameters $b$, to evaluate the performance of $\mathcal{C}(b)$. The function is defined as: $f(b)=D\left(\mathcal{C}(b) \rho \mathcal{C}(b)^{\dagger},|\mathbf{0}\rangle\langle\mathbf{0}|\right)=\operatorname{Tr}\left(|\mathbf{0}\rangle\langle\mathbf{0}| \cdot \mathcal{C}(b) \rho \mathcal{C}(b)^{\dagger}\right)$. The physical picture behind $f(b)$ is that it measures the overlap between the final state and the state $|\mathbf{0}\rangle\langle\mathbf{0}|$. This value can be easily obtained with applying the projective measurement operator $|\mathbf{0}\rangle\langle\mathbf{0}|$ in the computational basis.

Now, the key task is to solve a constrained optimization problem, that is, to find $b(t)$,

$$
\begin{aligned}
\max & f(b)=\operatorname{Tr}\left(|\mathbf{0}\rangle\langle\mathbf{0}| \cdot \mathcal{C}(b) \rho \mathcal{C}(b)^{\dagger}\right), \\
\text { s.t. } & \dot{\mathcal{C}}(b)=-i\left(\mathcal{H}_{0}+\mathcal{H}_{c}(b)\right) \mathcal{C}(b) .
\end{aligned}
$$

It is noted that, while this is a standard state-to-state optimal control problem, it can not be solved on a classical computer because the state $\rho$ is unknown. Besides, simulating the system's dynamics under $b(t)$ can be infeasible for a large quantum system. To circumvent the difficulty, we utilize the fact that the process of optimizing $\mathcal{C}(b)$ including the computing of the target function $f(b)$ and its gradient $g(b)=\nabla f(b)$ can be done with the controlled spin system itself [35]. On the other hand, a classical computer collects the gradient information from the experiments, stores the control parameters, determines the search direction, generates the next control sequence and feeds the iterate into the target system until the desired termination condition is fulfilled. As a result, such a QST process forms a closed loop, as illustrated in Fig. 1. More concretely, QST via HQC is divided into the following steps:

(i) For numerical optimization, we discretize the control sequence $b(t)$ by dividing it into $M$ slices

$$
b(t)=\left\{b_{x}[1], b_{x}[2], \ldots, b_{x}[M], b_{y}[1], b_{y}[2], \ldots, b_{y}[M]\right\} .
$$

The time length of each slice is a constant $\tau=T / M$ and the amplitude $b_{x, y}[m]$ in the $m$-th slice is also a constant. Then the propagator of the $m$-th slice is

$$
\mathcal{C}_{m}=\exp \left\{-i \tau\left(\mathcal{H}_{0}+b_{x}[m] \sum_{i=1}^{n} \sigma_{x}^{i}+b_{y}[m] \sum_{i=1}^{n} \sigma_{y}^{i}\right)\right\} .
$$

The total evolution of the sequence $b$ with $M$ slices can be described as $\mathcal{C}=\mathcal{C}_{M} \mathcal{C}_{M-1} \cdots \mathcal{C}_{2} \mathcal{C}_{1}$. Hence, the target function can be written as,

$$
f(b)=\operatorname{Tr}\left(|\mathbf{0}\rangle\langle\mathbf{0}| \cdot \mathcal{C}_{M} \cdots \mathcal{C}_{1} \rho \mathcal{C}_{1}^{\dagger} \cdots \mathcal{C}_{M}^{\dagger}\right) .
$$

(ii) A randomly generated set of pulse parameters $b_{x}^{(0)}[m]$ and $b_{y}^{(0)}[m]$ is chosen as the initial guess. Now we will describe two methods [29, 35] to calculate the gradient values $g_{x, y}[m]$. 


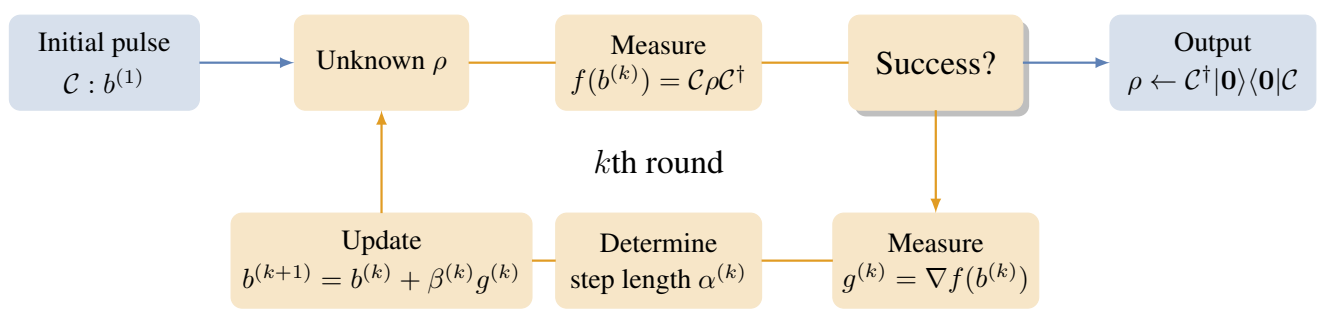

Figure 1. The workflow and schematic diagram of QST via HQC approach. The reconstruction of an unknown state $\rho$ is iteratively finished based on gradient-based searching. For each feed pulse $b^{(q)}$, the fitness function $f\left(b^{(q)}\right)$ and its gradient $g\left(b^{(q)}\right)=\nabla f\left(b^{(q)}\right)$ are computed by the system itself, while classical computer takes charge of the storage and update of the control pulse $b^{(q)}$ according to the $f$ and $g$. When the function $f$ reaches the desired accuracy, the optimal control pulse $b$ is obtained and is used to reconstruct $\rho$.

Method 1.-As long as the duration $\tau$ is small enough, the gradient value of the $m$-th slice $g_{\alpha}[m]=\partial f / \partial b_{\alpha}[m]$ with $\alpha=x, y$ can be approximately computed as

$$
g_{\alpha}[m]=\sum_{i=1}^{n} \operatorname{Tr}\left(-i \tau|\mathbf{0}\rangle\langle\mathbf{0}| \cdot \mathcal{C}_{m+1}^{M}\left[\sigma_{\alpha}^{i}, \mathcal{C}_{1}^{m} \rho \mathcal{C}_{1}^{m \dagger}\right] \mathcal{C}_{m+1}^{M \dagger}\right) .
$$

Here, $\mathcal{C}_{m+1}^{M}=\mathcal{C}_{M} \cdots \mathcal{C}_{m+2} \mathcal{C}_{m+1}$ and $\mathcal{C}_{1}^{m}=\mathcal{C}_{m} \cdots \mathcal{C}_{2} \mathcal{C}_{1}$. For any state $\rho$, one can check that there is

$$
\left[\sigma_{\alpha}^{i}, \rho\right]=i\left[\mathcal{R}_{\alpha}^{i}\left(\frac{\pi}{2}\right) \rho \mathcal{R}_{\alpha}^{i}\left(\frac{\pi}{2}\right)^{\dagger}-\mathcal{R}_{\alpha}^{i}\left(-\frac{\pi}{2}\right) \rho \mathcal{R}_{\alpha}^{i}\left(-\frac{\pi}{2}\right)^{\dagger}\right],
$$

where $\mathcal{R}_{\alpha}^{i}( \pm \pi / 2)$ is the $\pm \pi / 2$ rotation around $\alpha$ axis acting on the $i$-th qubit. If we define the notations $\mathcal{C}_{ \pm \alpha}^{i m}=$ $\mathcal{C}_{M} \ldots \mathcal{C}_{m+1} \mathcal{R}_{\alpha}^{i}( \pm \pi / 2) \mathcal{C}_{m} \ldots \mathcal{C}_{2} \mathcal{C}_{1}$, we can obtain the expression for $g_{\alpha}[m]$ :

$$
\tau \sum_{i=1}^{n}\left\{\operatorname{Tr}\left(|\mathbf{0}\rangle\langle\mathbf{0}| \cdot \mathcal{C}_{+\alpha}^{i m} \rho \mathcal{C}_{+\alpha}^{i m \dagger}\right)-\operatorname{Tr}\left(|\mathbf{0}\rangle\langle\mathbf{0}| \cdot \mathcal{C}_{-\alpha}^{i m} \rho \mathcal{C}_{-\alpha}^{i m \dagger}\right)\right\} .
$$

The two terms in the above equation are similar to that in the target function $f(b)$ in Eq. (3), because $\mathcal{C}_{ \pm \alpha}^{i m}$ is created by simply inserting a local operation $\mathcal{R}_{\alpha}^{i}( \pm \pi / 2)$ between the $m$-th and the $(m+1)$-th slice in $b(t)$. Hence, the $m$-th gradient $g_{\alpha}[m]$ can be obtained from a quantum system itself by performing $2 n$ measurement experiments. In total, we need one experiment for measuring the target function $f$ and additionally $4 n M$ experiments for measuring the $2 M$-dimensional gradient vector $g$. The number of required experiments is thus linear with the number of qubits.

Method 2.- Finite-difference approximation. To estimate the gradient value of the $m$-th slice $g_{\alpha}[m]$, we directly change the $m$-th control parameter $b_{\alpha}[m]$ by a step size $\delta$ in forward direction and create a new control sequence $b: b_{\alpha}[m]+\delta$. We apply the sequence to the controlled system and then measure its corresponding target function value $f\left(b: b_{\alpha}[m]+\delta\right)$ in the same way as measuring $f(b)$. In the first-order approximation, the gradient value of the $m$-th slices $g_{\alpha}[m]$ can be written as

$$
g_{\alpha}[m]=\frac{f\left(b: b_{\alpha}[m]+\delta\right)-f\left(b: b_{\alpha}[m]\right)}{\delta} .
$$

The step size $\delta$ should be cautiously chosen to guarantee convergence. Usually, we can fix $\delta$ as a sufficiently small value compared with the range of $b$. In this method, we only need $2 M+1$ experiments to determine the gradient values and the target function, independent with the number of qubits.

It should be noted that for both methods, the value of $\tau$ has to be kept small. As such, if the coupling strengths between the different spins are larger, then the shorter total time of the control sequence is required, and accordingly smaller number of the slices $M$ would suffice.

(iii) Next, we determine the search direction and generate the control sequence for the next iteration. Suppose that the control sequence in the $k$-th iteration is $b^{(k)}=$ $\left\{b_{x}^{(k)}[m], b_{y}^{(k)}[m]\right\}(m=1, \ldots, M)$ and the measured gradient is $g^{(k)}=\left\{g_{x}^{(k)}[m], g_{y}^{(k)}[m]\right\}$. Then we can move along the search direction to the next iteration,

$$
b_{\alpha}^{(k+1)}[m]=b_{\alpha}^{(k)}[m]+\beta_{\alpha}^{(k)} \cdot g_{\alpha}^{(k)}[m] .
$$

We choose an appropriate step length $\beta^{(k)}$ to achieve the optimal increase of $f$ in the gradient direction. The steps (ii) and (iii) are repeated until the target function $f(b)$ achieves the desired goal or converges to a local extremum.

(iv) With the optimal sequence at hand, we thus obtain a representation of the unknown state $\rho$ in terms of a parameterized control sequence. This is in analogy with that in variational quantum algorithms, a quantum state is expressed in terms of a parameterized quantum circuit. In some circumstances, one can directly calculate out $\rho=\mathcal{C}(b)^{\dagger}|\mathbf{0}\rangle\langle\mathbf{0}| \mathcal{C}(b)$ efficiently. For example, when the system Hamiltonian is onedimensional, it is possible to simulate the dynamics $\mathcal{C}(b)^{\dagger}$ with high accuracy by means of tensor-network based techniques like the time-dependent density matrix renormalization group $[40,41]$. Our method thus provides the possibility to perform efficient QST for tasks in near-term quantum systems.

\section{Applications}

In exploiting the potential applications of our proposed HQC method, one important problem is the scaling issue. Generally, due to the intrinsic complexity of the state tomography problem, it is unlikely to have a tomographic scheme with favorable scaling for any quantum state from the Hilbert space. What one can do in practice is to presume a certain class of quantum states, and then to make a tomographic 
scheme feasible so that it can replace the inefficient full state tomography approach. This is meaningful because the states involved in common experiments do not spread in the whole Hilbert space. Actually, in principle most quantum states are exponentially hard to reach or even to approximate, so most states are in fact beyond the grasp of the quantum experimenters $[1,42]$. Therefore, the target states to be reconstructed should only locate in a part of the Hilbert space. The scaling issue of our HQC method has to be comprehended under this general picture. That is, without any restriction of the target state, the number of slices required for a control sequence to drive it to $|\mathbf{0}\rangle\langle\mathbf{0}|$ tends to be exponentially large. The actual problem of interest is to find out whether HQC provides better scaling for a certain class of quantum states.

As an applicative example, we show that the QST via HQC approach can reconstruct dynamical states of quantum manybody systems. Consider the scenario shown in Fig. 2(a) where we attempt to tomography a dynamical state of a quantum many-body system. There have been remarkable theoretical findings by researchers in many-body physics and quantum information theory showing that, dynamical states of quantum many-body systems can usually be described by only a polynomial number of parameters [43]. It is reasonable to assume that, such states have polynomially scaled complexity, even for the case of a chaotic many-body Hamiltonian. For example, a latest work has proved that the complexity of the quantum state generated by a local random quantum circuit grows linearly for a long time [39]. Previously it has been demonstrated that, to estimate dynamical states of quantum many-body systems, the machine learning approach can be a simple substitute for full state tomography [44, 45]. Here, we numerically simulate the potential of using our method for reconstructing the dynamical states of a quantum many-body

(a)

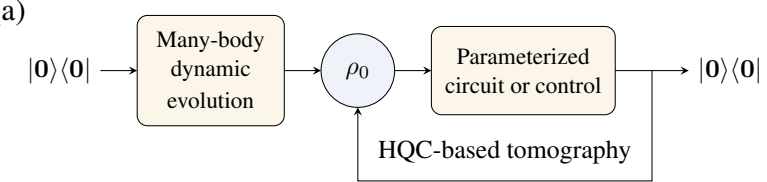

(b)

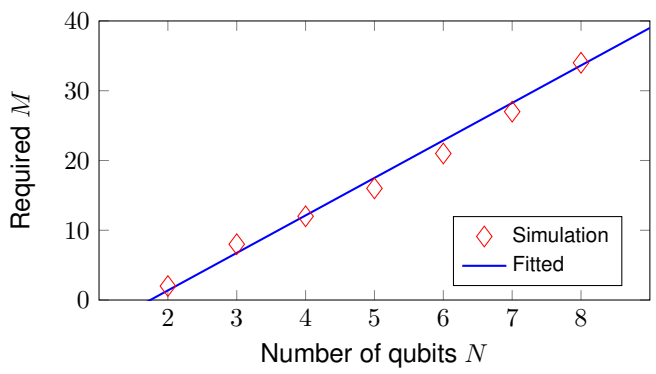

Figure 2. (a) Tomography of a dynamical state of a many-body system using the HQC approach. (b) The number of slices $M$ required against the size of the system. The target state is a time-evolved state at some time $T: \rho_{0}=|\phi(T)\rangle\langle\phi(T)|$ with $|\phi(T)\rangle=e^{-i \mathcal{H}_{0} T}|\mathbf{0}\rangle$. In the simulation, we set $T=3 \mathrm{~s}$ and the size of the system ranges from 2 to 8 , and then we seek the minimum number of slices required for the target state $|\phi(T)\rangle$ to be driven towards $|\mathbf{0}\rangle\langle\mathbf{0}|$ with fidelity over $99 \%$. The line is the fitting result over these points. system and then present an experimental test in the next section.

To specify the problem, we consider a quantum many-body system which starts from the initial state $|\mathbf{0}\rangle\langle\mathbf{0}|$ and evolves to the final dynamical state $\rho_{0}$ after a polynomial evolution time $t$. Suppose that just the state $\rho$ is given to us, with no more information of the Hamiltonian and $t$. Despite this, we can be confident about the existence of an efficient sequence capable of realizing the state transfer between $|\mathbf{0}\rangle\langle\mathbf{0}|$ and $\rho$. For example, the reverse of the transfer can be realized simply by reversing the many-body Hamiltonian, which can be simulated via a parameterized quantum circuit or a control sequence. Consequently, although the actual parameters found via our hybrid optimization method may not be exactly the same as the said reverse evolution parameters, the number of parameters can keep polynomially scaled with the size of system. To support the statement, here we choose the reconstruction of the dynamical states of the Ising-model Hamiltonian as an illustrative example and numerically simulate how the number of parameters required scales with the size of the system using our HQC approach. In our simulation, the Ising Hamiltonian is

$$
\mathcal{H}_{\text {Ising }}=-\sum_{i=1}^{n-1} \sigma_{i}^{z} \sigma_{i+1}^{z}+\sum_{i=1}^{n} \sigma_{i}^{x}
$$

and the control Hamiltonian takes the form $\sum_{i=1}^{n} h_{i}^{(m)} \sigma_{i}^{x}+$ $\sum_{i=1}^{n} b_{i}^{(m)} \sigma_{i}^{y}$, where $h_{i}^{(m)}$ and $b_{i}^{(m)}$ are the control parameters of the $m$-th slice on the site $i$. From the results in Fig. 2(b), it is evident that the HQC approach scales well with the size of the system.

\section{EXPERIMENT}

\section{A. System}

As a proof-of-principle demonstration, we experimentally test the feasibility of our method by reconstructing two kinds of quantum states on a 4-qubit NMR simulator [46-48]. As shown in Fig. 3(a), the spins we used are carbon nuclei in ${ }^{13} \mathrm{C}$-labeled trans-crotonic acid dissolved in d6-acetone after decoupling them from the methyl group $\mathrm{M}$ and the hydrogen atoms. Our experiments were carried out on a Bruker $600 \mathrm{MHz}$ spectrometer at room temperature $298 \mathrm{~K}$. Under the weak coupling approximation, the Hamiltonian of our system in reference frame can be written as,

$$
\mathcal{H}_{\mathrm{int}}=\sum_{j=1}^{4} \pi \nu_{j} \sigma_{z}^{j}+\sum_{j<k,=1}^{4} \frac{\pi}{2} J_{j k} \sigma_{z}^{j} \sigma_{z}^{k}
$$

with the chemical shifts $\nu_{j}$ and the J-coupling strengths $J_{j k}$, respectively. Figure 3(a) gives the molecular structure and the physical parameters. 


\section{B. Scheme}

The experimental procedure can be divided into four parts: (i) Initialize the spins into the initial state $|0000\rangle$; (ii) Prepare the target state $\rho$ from $|0000\rangle$; (iii) At the $k$ th iteration, feed the pulse $b^{(k)}$ into the spin system and measure the fitness function and its gradient; (iv) Update the pulse for the next iteration and perform the iterations until achieving the goal.

First, we initialized the NMR system to a pseudo-pure state (PPS) from the thermal equilibrium state which is a highly mixed state. This was implemented using the spatial averaging technique involving unitary operations and gradient fields $[49,50]$. The density matrix of PPS is of the form $\rho_{\mathrm{g}}=$ $(1-\epsilon) \mathbb{I} / 16+\epsilon|0000\rangle\langle 0000|$, here $\epsilon \approx 10^{-5}$ represents the polarization and $\mathbb{I}$ the $16 \times 16$ identity operator. In the following, we just use the part $|0000\rangle\langle 0000|$ (PPS) as the description of the spins, while ignoring the identity part, because the identity part of the state does not evolve nor contribute to the NMR signals under any unitary operations [51]. Our experimentally prepared PPS achieved over 0.99 fidelity according to the state fidelity definition $F(\rho, \sigma)=\operatorname{Tr}(\rho \sigma) / \sqrt{\operatorname{Tr}\left(\rho^{2}\right)} \sqrt{\operatorname{Tr}\left(\sigma^{2}\right)}$ $[52,53]$.

Second, we prepared the following target states and then reconstructed them via HQC-based QST.

(1) Dynamical state of a quantum many-body system. We choose to reconstruct the dynamical states of a prototypical quantum many-body system, namely the transverse field Ising-model. As an illustrative example, we consider the

(a)
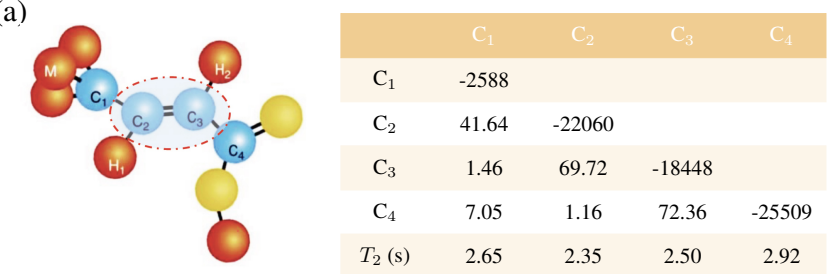

(b)
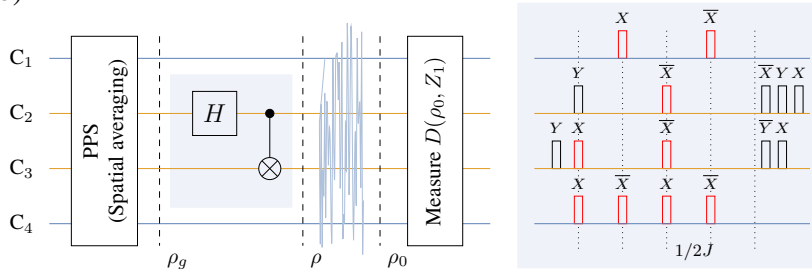

Figure 3. Molecular parameters and experimental quantum circuit. (a) Molecular structure and Hamiltonian parameters for ${ }^{13} \mathrm{C}$-labeled trans-crotonic acid. The table shows the chemical shifts and $\mathrm{J}$ couplings in the diagonal and off-diagonal elements, respectively. $\mathrm{C}_{2}$ and $\mathrm{C}_{3}$ have the maximum coupling value of $72.36 \mathrm{~Hz}$. (b) Quantum circuit for testing the proposed method and NMR pulse sequence for preparing $\rho$. It includes three parts: PPS initialization, the preparation for the target state $\rho$, and the pulse optimization by quantum system itself. Hadamard and CNOT gates can also be realized by a pulse sequence including local rotations and J-coupling evolutions illustrated in the right of (b), where the black and red rectangles represent $\pi / 2$ and $\pi$ pulses around the directions indicated on top of them, respectively.
Hamiltonian $\mathcal{H}_{\text {Ising }}$ in Eq. (7). The target state is a timeevolved state $|\phi(T)\rangle=e^{-i \mathcal{H}_{\text {lsing } T}}|\mathbf{0}\rangle$ at time $T=0.6 \mathrm{~s}$. In experiment, to prepare $|\phi(T)\rangle$, a $3 \mathrm{~ms}$ optimized radiofrequency (RF) pulse with fidelity 0.995 is applied to the system. This shaped pulse has been designed to be robust to RF inhomogeneity.

(2) Entangled quantum state. We prepare the spins $\mathrm{C}_{2}$ and $\mathrm{C}_{3}$ to an entangled state $(|00\rangle+|11\rangle) / \sqrt{2}$ and keep the other spins stay in the $|0\rangle$ state. This can be done via a quantum circuit involving a Hadamard gate on $\mathrm{C}_{2}$ and a controlled-NOT gate $\mathrm{CNOT}_{2,3}$. In NMR, the Hadamard gate can be decomposed as $\mathcal{R}_{x}^{2}(\pi) \mathcal{R}_{y}^{2}(\pi / 2)$ and $\mathrm{CNOT}_{2,3}$ can be decomposed as

$$
\mathrm{CNOT}_{2,3}=\sqrt{i} \mathcal{R}_{z}^{2}\left(\frac{\pi}{2}\right) \mathcal{R}_{z}^{3}\left(-\frac{\pi}{2}\right) \mathcal{R}_{x}^{3}\left(\frac{\pi}{2}\right) \mathcal{U}\left(\frac{1}{2 J}\right) \mathcal{R}_{y}^{3}\left(\frac{\pi}{2}\right),
$$

Here, $\mathcal{R}_{z}^{2}(\pi / 2)$ and $\mathcal{R}_{z}^{3}(-\pi / 2)$ can be written as

$$
\begin{aligned}
\mathcal{R}_{z}^{2}\left(\frac{\pi}{2}\right) & =\mathcal{R}_{x}^{2}\left(\frac{\pi}{2}\right) \mathcal{R}_{y}^{2}\left(\frac{\pi}{2}\right) \mathcal{R}_{x}^{2}\left(-\frac{\pi}{2}\right), \\
\mathcal{R}_{z}^{3}\left(-\frac{\pi}{2}\right) & =\mathcal{R}_{x}^{3}\left(\frac{\pi}{2}\right) \mathcal{R}_{y}^{3}\left(-\frac{\pi}{2}\right) \mathcal{R}_{x}^{3}\left(-\frac{\pi}{2}\right) .
\end{aligned}
$$

The evolution operator $\mathcal{U}(1 / 2 J)$ represents the $J$-coupling evolution $e^{-i \pi \sigma_{z}^{2} \sigma_{z}^{3} / 4}$ and it can be realized by inserting refocusing pulses,

$$
\begin{aligned}
& \mathcal{R}_{x}^{2,3,4}(\pi) \rightarrow f\left(\frac{1}{8 J}\right) \rightarrow \mathcal{R}_{x}^{1}(\pi) \mathcal{R}_{x}^{4}(-\pi) \rightarrow f\left(\frac{1}{8 J}\right) \\
& \rightarrow \mathcal{R}_{x}^{2,3}(-\pi) \mathcal{R}_{x}^{4}(\pi) \rightarrow f\left(\frac{1}{8 J}\right) \rightarrow \mathcal{R}_{x}^{1,4}(-\pi) \rightarrow f\left(\frac{1}{8 J}\right),
\end{aligned}
$$

where $f(1 / 8 J)$ represents the system free evolution of duration $1 / 8 J$, and $\mathcal{R}_{\alpha}^{i}(\theta)$ is a rotation about the axis $\alpha=x$ or $y$ with angle $\theta$ acting on the $i$-th qubit. The circuit and the corresponding pulse sequence is shown in Fig. 3(b).

After the above state preparation step, a 4-qubit full QST was implemented. The QST results show that the prepared dynamical state and the entangled state has the experimental fidelity of 0.99 and 0.98 , respectively. The purpose of doing the conventional full QST is for verifying that the target state has indeed been prepared and also for the subsequent comparison with our method. The basic principle of QST in NMR is as follows [54]. NMR measurement is essentially ensemble measurement, one can determine the expectation value of an observable using one experiment provided that the signal-tonoise ratio is good. The NMR setup measures the expectation values of the single-quantum coherence operators. If one wants to measure other operators, readout pulses are needed before data acquisition to transfer them to detectable singlequantum coherences. Finally, all the measurement data are collected to infer the coefficients of $\rho$.

Next, following our proposed HQC method, we iteratively optimize a control sequence $b(t)$ to drive the target state towards $|0000\rangle\langle 0000|$. We start from an initial guess $b^{(1)}=$ $\left\{b_{x}^{(1)}, b_{y}^{(1)}\right\}$. When it proceeds to the $k$-th iteration we fed the optimized pulse $b^{(k)}$ into the NMR system, and then estimated the resulting fitness function $D\left(\rho_{0}^{(k)}, Z_{1}\right)$ from measuring the expectation value of the operator $Z_{1}=\sigma_{z} \otimes$ 
$|000\rangle\langle 000|$. Notice that, optimizing $Z_{1}$ is equivalent to optimizing $D\left(\rho_{0}^{(k)},|0000\rangle\langle 0000|\right)$, and importantly, it can be done with just implementing a single $\pi / 2$ readout pulse on the first spin $\mathrm{C}_{1}$.

Last, we changed the control parameters $b_{x}^{(k)}[m]$ and $b_{y}^{(k)}[m]$ by a step size $\triangle=1 \mathrm{kHz}$ for the $m$-th slice to measure the gradient values $g_{x}^{(k)}[m]$ and $g_{y}^{(k)}[m]$. Here, (i) for the dynamical state, we set the number of slices as $M=125$ and the time length for each slice as $\tau=40 \mu$ s for the initial pulse; (ii) for the entangled state, we set the number of slices as $M=150$ and the duration of each slice as $\tau=60 \mu$ s. So there are in total $2 \times 125=250$ control parameters to be optimized in each iteration for the dynamical state (300 control parameters for the case of entangled state). After the gradient vector $g^{(k)}$ was determined in experiments, we updated the pulse along the gradient direction for obtaining an increment of $D\left(\rho_{0}^{(k+1)}, Z_{1}\right)$. Here, $2 M$ experiments are necessary to determine the gradient values for one round of iteration.

After the optimization was finished, we also performed 4qubit QST on the final state to evaluate its fidelity with the ideal state $\rho_{0}=|0000\rangle\langle 0000|$. We use $\rho_{0}^{\mathrm{e}}$ to denote the experimentally reconstructed final state. We found that the fidelity between $\rho_{0}^{\mathrm{e}}$ and the ideal state $\rho_{0}$ is 0.986 for the dynamical state case and 0.943 for the entangled state case. This means that $\rho$ was almost driven into $|0000\rangle\langle 0000|$ through the optimized pulse.

\section{Results}

Now we present the results of the reconstruction via HQCbased QST for the dynamical state and the entangle state.

The dynamical state.-In this experiment, we in total performed 6 iterations and 250 experiments for each iteration such that the target state $\rho$ was reconstructed with sufficient quality. In Fig. 4(a), it is clear that the fitness function $D\left(\rho_{0}, Z_{1}\right)$ and the fidelity of the state $\rho^{(k)}$ are approaching the optimal value of 1 with the increasing number of iterations, and there is a good agreement between the experimentally measured and simulated $D\left(\rho_{0}, Z_{1}\right)$. Here, $\rho^{(k)}$ is the reconstructed state via our method at the $k$-th iteration, which is computed as $\mathcal{C}\left(b^{(k)}\right)^{\dagger}|0000\rangle\langle 0000| \mathcal{C}\left(b^{(k)}\right)$ via the pulse $b^{(k)}$ on the NMR simulator. The real parts of the density matrices $\rho^{(k)}$ for $k=1,2,3$, and 6 are presented in Fig. 4(b). Eventually, over 0.97 fidelity was achieved via our method in reconstructing the dynamical state. In each iteration, we measured the gradient vector $g^{(k)}$, whose precision determines the search direction of the optimization and hence the performance of the next iteration. Here, we place the comparison between the measured and simulated gradient vector $g_{x}^{(1)}$ in Fig. 4(e).

The entangled state.-The results are shown in Fig. 4(c) and 4(d). Figure 4(c) presents the simulated and the experimentally measured $D\left(\rho_{0}, Z_{1}\right)$, as well as the fidelity of $\rho^{(k)}$ as a function of the iteration number $k$. Here, the simulated $D\left(\rho_{0}, Z_{1}\right)$ was directly computed by applying the iterated pulse $b^{(k)}$ on the ideal state $\rho$ and numerically mea- suring the expectation value of $Z_{1}$ on the classical computer. $D\left(\rho_{0}, Z_{1}\right)$ and $D\left(\rho_{0},|0000\rangle\langle 0000|\right)$ can be both considered as the fitness function in this optimization. If $D\left(\rho_{0}, Z_{1}\right)$ converges to the optimal value, then so does the fitness function $D\left(\rho_{0},|0000\rangle\langle 0000|\right)$. As shown in Fig. 4(c), $D\left(\rho_{0}, Z_{1}\right)$ increased to 0.90 after 4 iterations, and then reached 0.95 when iteration number $k=6$. Similarly, we estimated the quality of the reconstructed state $\rho^{(k)}$ in each iteration, by applying the $k$ th iterate pulse $b^{(k)}$ on the state $|0000\rangle\langle 0000|$. Figure $4(d)$ presents the real parts of the density matrices of the reconstructed states $\rho^{(k)}$ for iteration number $k=1,2,3$, and 6. It obviously shows that the state $\rho^{(k)}$ is approaching the ideal state in experiments, and the density matrix form of Bell state between $\mathrm{C}_{2}$ and $\mathrm{C}_{3}$ shows up when $k=5$. Finally, we successfully realized the reconstruction of the entangled state via our method with around 0.95 fidelity. In each iteration, the gradient vector $g^{(k)}$ with 300 parameters was measured on the NMR simulator. Figure 4(f) presents the measured and simulated gradients for the first iteration.

In the NMR spectrum, each qubit's signal contains 8 peaks because of its coupling with the other three spins [55]. If a $\pi / 2$ readout pulse acting on the first spin is applied on the ideal state $|0000\rangle$, there should appear a single-peak signal labeled by the rest spins $|000\rangle$. Figure 5 shows the spectra of $C_{1}$ as a function of iteration number $k$. Single-peak appearing after $k=4$ also implied that convergence was almost achieved.

\section{Error Analysis and Convergence}

In experiments, there are certain error sources including the imperfections of PPS initialization, the infidelities of the GRAPE pulses, and decoherence effects. These error sources have consequences in the experimental results, which we describe as follows. (i) They cause a deviation between the experimental and the ideal results. Here, in order to estimate the influence of the potential errors from real experiments on the values $D\left(\rho_{0}, Z_{1}\right)$, we numerically simulated the quantum dynamics which starts from the prepared state in experiments and evolves under the GRAPE pulse with consideration of a decoherence model for each iteration. We further compared the simulated values $D_{\text {sim }}$ with the ideal ones $D_{\text {th }}$, and then computed the standard deviation of our simulated results as $\epsilon=\sqrt{\sum_{i}^{K}\left(D_{\text {sim }}^{i}-D_{\mathrm{th}}^{i}\right)^{2} /(K-1)}$, with $K$ the number of iterations. It is found that $\epsilon$ is $2.45 \%$ for the dynamical state, and is $4.96 \%$ for the entangled state. (ii) They lead to the inaccuracies in the measurement of the gradients. Imperfections in measuring gradients deviate the search direction from the desired one and could cause the search be trapped by local extremum. (iii) Minor experimental errors can lead to inaccuracies of the gradients and the impurity of $\rho^{e}$. In that case, it is impossible to get a unitary sequence $\mathcal{C}(b)$ that can perfectly drive the mixed state $\rho^{e}$ to the pure state $|\mathbf{0}\rangle\langle\mathbf{0}|$. This explains why our result in practice did not converge to the perfect fidelity of 1. 


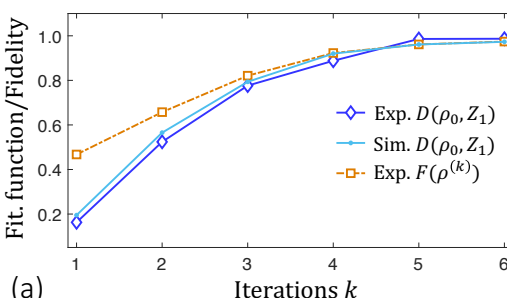

(a)

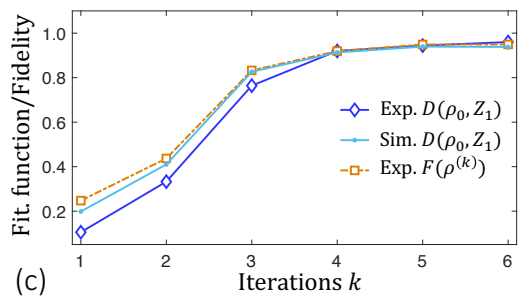

(c)

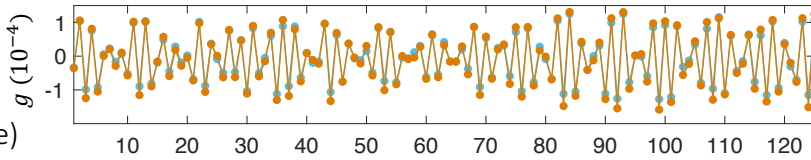

(b) $\operatorname{Real}\left(\rho^{(1)}\right), F=0.47$

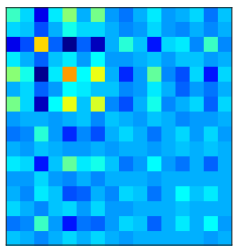

(d) $\operatorname{Real}\left(\rho^{(1)}\right), F=0.28$
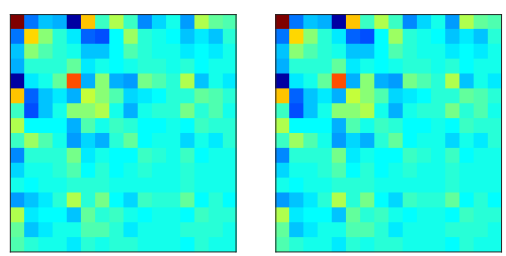

$\operatorname{Real}\left(\rho^{(2)}\right), F=0.66$

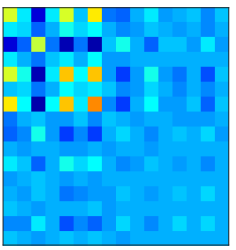

$\operatorname{Real}\left(\rho^{(2)}\right), F=0.44$

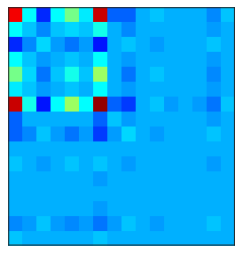

$\operatorname{Real}\left(\rho^{(3)}\right), F=0.83$

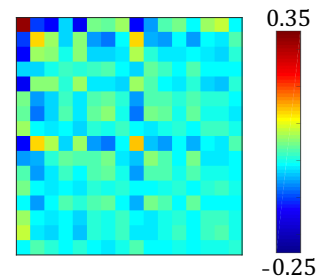

$\operatorname{Real}\left(\rho^{(6)}\right), F=0.97$

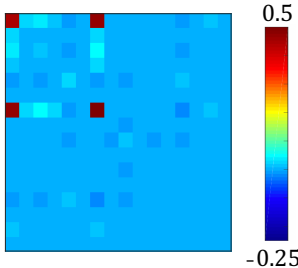

$\operatorname{Real}\left(\rho^{(6)}\right), F=0.95$

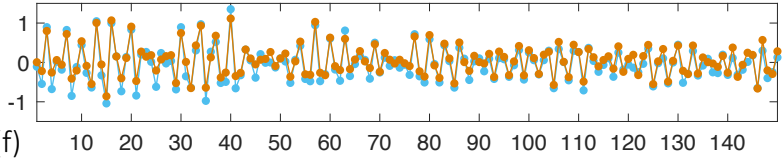

Figure 4. Experimental results in the reconstruction of $\rho$ by variational HQC-based QST. (a)-(b) The result for the dynamical state. (c)-(d) The result for the entangled state. (a) and (c) The fitness function and the fidelity as a function of iteration number $k$. The cyan and blue points represent the simulated $D\left(\rho_{0}, Z_{1}\right)$ and the experimentally measured $D\left(\rho_{0}, Z_{1}\right)$ using the pulse $b^{(k)}$ on the controlled system. $F\left(\rho^{(k)}\right)$ is the fidelity of the reconstructed state $\rho^{(k)}$ with the target state. (b) and (d) The real parts of the density matrix elements of the reconstructed $\rho^{(k)}$ for $k=1,2,3$, and 6 for the dynamical state and the entangled state. They were estimated from $\mathcal{C}\left(b^{(k)}\right)^{\dagger}|0000\rangle\langle 0000| \mathcal{C}\left(b^{(k)}\right)$ by implementing the pulse $b^{(k)}$ on the state $|0000\rangle$. (e) and (f) The gradient vectors $g_{x}^{(1)}$ for the case of the dynamical state and entangled state, respectively. The experimental and simulated data are labeled by the orange and the cyan, respectively.
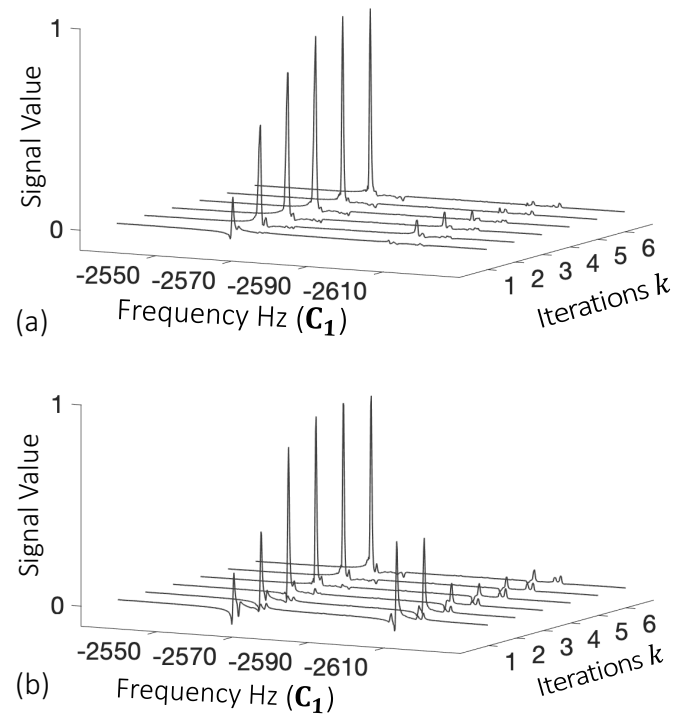

Figure 5. Experimental spectra of the spin $\mathrm{C}_{1}$ for each iteration for the dynamical state (a) and the entangled state (b). The spectra were obtained by performing a $\pi / 2$ readout pulse on the spin $\mathrm{C}_{1}$ before data acquisition. Single-peak pattern appeared after $k=3$, and the intensity of this peak increased to about 0.9 after $k=4$.

\section{CONCLUSION}

We presented a self-learning QST method via the variational HQC approach, in which the fitness function and gradients are measured online with the system itself. Hence, such a method can reconstruct the real states in experiments with the advantages of reliability and efficiency. While the conventional brute-force full state tomography approach needs to perform exponential number of measurements, our method is promising for provide a better scaling for a broad class of practical quantum states, e.g., dynamical states of a quantum many-body system. Another advantage is that, unlike conventional QST methods that are based on raw experimental measurement data, which usually output unphysical density matrices (e.g., not positive semi-definite) and thus require some subsequent correction techniques like maximum likelihood estimation [56], the HQC method directly outputs the valid density matrix once the desired control sequence is found. Therefore, our work offers a new way to enhance the efficiency of QST in practice.

The variational HQC-based QST method can be applied to but not limited to reconstruction of the dynamical states of quantum many-body systems, the ground states of $k$-local Hamiltonians in quantum statistical mechanics, and the entangled quantum states commonly used in quantum computing and quantum communication. Undoubtedly, these states 
are frequently encountered in quantum experiments and are of importance for emerging quantum technologies. Many recent known research work have been devoted to the tomography problem for these quantum states [25, 44, 45, 57-59]. Our variational HQC method offers an alternative choice. Using techniques of NMR, we have experimentally demonstrated the feasibility of performing QST via our method by successfully reconstructing a 4-qubit dynamical state of quantum manybody system and an entangled state. For future studies, it would be interesting to extend the current method to other experimental platforms. We expect the methodology developed here can become a useful tool for practical quantum tomography on intermediate-scale quantum devices that are about to appear in the near term.

\section{ACKNOWLEDGMENTS}

T. X., J. L. and D. L. are supported by the National Key Research and Development Program of China (Grants No. 2019YFA0308100), National Natural Science Foundation of China (Grants No. 11605005, No. 11875159, No. U1801661, No. 11905099, and No. 11975117), Science, Technology and Innovation Commission of Shenzhen Municipality (Grants No. ZDSYS20170303165926217 and No. JCYJ20170412152620376), Guangdong Innovative and Entrepreneurial Research Team Program (Grant No. 2016ZT06D348), Guangdong Basic and Applied Basic Research Foundation (Grant No. 2019A1515011383).
[1] M. A. Nielsen and I. L. Chuang, Quantum Computation and Quantum Information (Cambridge University Press, Cambridge, 2000).

[2] A. I. Lvovsky and M. G. Raymer, Rev. Mod. Phys. 81, 299 (2009).

[3] M. Baur, A. Fedorov, L. Steffen, S. Filipp, M. Da Silva, and A. Wallraff, Phys. Rev. Lett. 108, 040502 (2012).

[4] M. Hofheinz, H. Wang, M. Ansmann, R. C. Bialczak, E. Lucero, M. Neeley, A. O'connell, D. Sank, J. Wenner, J. M. Martinis, and A. N. Cleland, Nature 459, 546 (2009).

[5] T. Jullien, P. Roulleau, B. Roche, A. Cavanna, Y. Jin, and D. Glattli, Nature 514, 603 (2014).

[6] M. Cramer, M. B. Plenio, S. T. Flammia, R. Somma, D. Gross, S. D. Bartlett, O. Landon-Cardinal, D. Poulin, and Y.-K. Liu, Nat. Commun. 1, 149 (2010).

[7] D. Gross, Y.-K. Liu, S. T. Flammia, S. Becker, and J. Eisert, Phys. Rev. Lett. 105, 150401 (2010).

[8] S. T. Flammia and Y.-K. Liu, Phys. Rev. Lett. 106, 230501 (2011).

[9] T. Baumgratz, D. Gross, M. Cramer, and M. B. Plenio, Phys. Rev. Lett. 111, 020401 (2013).

[10] C. Ferrie, Phys. Rev. Lett. 113, 190404 (2014).

[11] B. P. Lanyon, C. Maier, M. Holzäpfel, T. Baumgratz, C. Hempel, P. Jurcevic, I. Dhand, A. Buyskikh, A. Daley, M. Cramer, M. B. Plenio, R. Blatt, and C. F. Roos, Nat. Phys. 13, 1158 (2017).

[12] R. J. Chapman, C. Ferrie, and A. Peruzzo, Phys. Rev. Lett. 117, 040402 (2016).

[13] D. Ahn, Y. S. Teo, H. Jeong, F. Bouchard, F. Hufnagel, E. Karimi, D. Koutnỳ, J. Řeháček, Z. Hradil, G. Leuchs, and L. L. Sánchez-Soto, Phys. Rev. Lett. 122, 100404 (2019).

[14] D. Mahler, L. A. Rozema, A. Darabi, C. Ferrie, R. BlumeKohout, and A. Steinberg, Phys. Rev. Lett. 111, 183601 (2013).

[15] R. Okamoto, M. Iefuji, S. Oyama, K. Yamagata, H. Imai, A. Fujiwara, and S. Takeuchi, Phys. Rev. Lett. 109, 130404 (2012).

[16] F. Huszár and N. M. Houlsby, Phys. Rev. A 85, 052120 (2012).

[17] T. Xin, D. Lu, J. Klassen, N. Yu, Z. Ji, J. Chen, X. Ma, G. Long, B. Zeng, and R. Laflamme, Phys. Rev. Lett. 118, 020401 (2017).

[18] N. Wyderka, F. Huber, and O. Gühne, Phys. Rev. A 96, 010102 (2017).

[19] P. Parashar and S. Rana, Phys. Rev. A 80, 012319 (2009).

[20] J. Chen, H. Dawkins, Z. Ji, N. Johnston, D. Kribs, F. Shultz, and B. Zeng, Phys. Rev. A 88, 012109 (2013).
[21] G. Torlai, G. Mazzola, J. Carrasquilla, M. Troyer, R. Melko, and G. Carleo, Nat. Phys. 14, 447 (2018).

[22] X. Gao and L.-M. Duan, Nat. Commun. 8, 662 (2017).

[23] M. Kieferová and N. Wiebe, Phys. Rev. A 96, 062327 (2017).

[24] J. Gao, L.-F. Qiao, Z.-Q. Jiao, Y.-C. Ma, C.-Q. Hu, R.-J. Ren, A.-L. Yang, H. Tang, M.-H. Yung, and X.-M. Jin, Phys. Rev. Lett. 120, 240501 (2018).

[25] T. Xin, S. Lu, N. Cao, G. Anikeeva, D. Lu, J. Li, G. Long, and B. Zeng, npj Quantum Inf. 5 (2019).

[26] B. Qi, Z. Hou, L. Li, D. Dong, G. Xiang, and G. Guo, Sci. Rep. 3, 3496 (2013).

[27] B. Bauer, D. Wecker, A. J. Millis, M. B. Hastings, and M. Troyer, Phys. Rev. X 6, 031045 (2016).

[28] S. Bravyi, G. Smith, and J. A. Smolin, Phys. Rev. X 6, 021043 (2016).

[29] J. R. McClean, J. Romero, R. Babbush, and A. Aspuru-Guzik, New J. Phys. 18, 023023 (2016).

[30] D. Wecker, M. B. Hastings, and M. Troyer, Phys. Rev. A 92, 042303 (2015).

[31] Y. Li and S. C. Benjamin, Phys. Rev. X 7, 021050 (2017).

[32] A. Arrasmith, L. Cincio, A. T. Sornborger, W. H. Zurek, and P. J. Coles, Nat. Commun. 10, 3438 (2019).

[33] A. Kandala, A. Mezzacapo, K. Temme, M. Takita, M. Brink, J. M. Chow, and J. M. Gambetta, Nature 549, 242 (2017).

[34] A. Peruzzo, J. McClean, P. Shadbolt, M.-H. Yung, X.-Q. Zhou, P. J. Love, A. Aspuru-Guzik, and J. L. O'brien, Nat. Commun. 5, 4213 (2014).

[35] J. Li, X. Yang, X. Peng, and C.-P. Sun, Phys. Rev. Lett. 118, 150503 (2017).

[36] J. R. McClean, M. E. Kimchi-Schwartz, J. Carter, and W. A. de Jong, Phys. Rev. A 95, 042308 (2017).

[37] P. D. Johnson, J. Romero, J. Olson, Y. Cao, and A. AspuruGuzik, arXiv:1711.02249.

[38] R. LaRose, A. Tikku, É. O’Neel-Judy, L. Cincio, and P. J. Coles, npj Quant. Inf. 5, 8 (2019).

[39] F. G. Brandão, W. Chemissany, N. Hunter-Jones, R. Kueng, and J. Preskill, arXiv:1912.04297.

[40] S. R. White and A. E. Feiguin, Phys. Rev. Lett. 93, 076401 (2004).

[41] U. Schollwöck, Rev. Mod. Phys. 77, 259 (2005).

[42] D. Poulin, A. Qarry, R. Somma, and F. Verstraete, Phys. Rev. Lett. 106, 170501 (2011).

[43] D. Perez-Garcia, F. Verstraete, M. M. Wolf, and J. I. Cirac, Quant. Inf. Comp. 7, 401 (2007). 
[44] B. P. Lanyon, C. Maier, M. Holzäpfel, T. Baumgratz, C. Hempel, P. Jurcevic, I. Dhand, A. S. Buyskikh, A. J. Daley, M. Cramer, M. B. Plenio, R. Blatt, and C. F. Roos, Nat. Phys. 13, 1158 (2017).

[45] G. Torlai, G. Mazzola, J. Carrasquilla, M. Troyer, R. Melko, and G. Carleo, Nat. Phys. 14, 447 (2018).

[46] T. Xin, B.-X. Wang, K.-R. Li, X.-Y. Kong, S.-J. Wei, T. Wang, D. Ruan, and G.-L. Long, Chinese Physics B 27, 020308 (2018).

[47] Z. Luo, J. Li, Z. Li, L.-Y. Hung, Y. Wan, X. Peng, and J. Du, Nat. Phys. 14, 160 (2018).

[48] X.-W. Yao, H. Wang, Z. Liao, M.-C. Chen, J. Pan, J. Li, K. Zhang, X. Lin, Z. Wang, Z. Luo, et al., Phys. Rev. X 7, 031041 (2017).

[49] D. G. Cory, M. D. Price, and T. F. Havel, Physica D: Nonlinear Phenomena 120, 82 (1998).

[50] D. G. Cory, A. F. Fahmy, and T. F. Havel, Proceedings of the National Academy of Sciences 94, 1634 (1997).

[51] E. Knill and R. Laflamme, Phys. Rev. Lett. 81, 5672 (1998).
[52] J.-S. Lee, Physics Letters A 305, 349 (2002).

[53] G. M. Leskowitz and L. J. Mueller, Phys. Rev. A 69, 052302 (2004).

[54] J. Li, S. Huang, Z. Luo, K. Li, D. Lu, and B. Zeng, Phys. Rev. A 96, 032307 (2017).

[55] T. Xin, S.-J. Wei, J. S. Pedernales, E. Solano, and G.-L. Long, Phys. Rev. A 96, 062303 (2017).

[56] H. Singh, Arvind, and K. Dorai, Physics Letters A 380, 3051 (2016).

[57] E. Bairey, I. Arad, and N. H. Lindner, Phys. Rev. Lett. 122, 020504 (2019).

[58] G. Torlai, B. Timar, E. P. van Nieuwenburg, H. Levine, A. Omran, A. Keesling, H. Bernien, M. Greiner, V. Vuletić, M. D. Lukin, R. G. Melko, and M. Endres, Phys. Rev. Lett. 123, 230504 (2019).

[59] M. Ohliger, V. Nesme, and J. Eisert, New Journal of Physics 15, 015024 (2013). 